\title{
Increase in MMP-9 Expressing CD11b+ cells in a CD44 Dependent Way Reduces Severity of Experimental Autoimmune Encephalomyelitis
}

\author{
Sujata Kar, ${ }^{1}$ Kalipada Kar $^{1}$ \\ ${ }^{1}$ Department of Physiology, College of Medical Sciences-Teaching Hospital, Chitwan, Nepal.
}

\begin{abstract}
Background: Multiple sclerosis (MS) is a dangerous neurodegenerative disorder. Various aspects of the disease have been studied in experimental animal model. Migration of immune cells to the central nervous system (CNS) is a predominant feature of MS. CD44 molecule has been reported to be involved in many important biological processes including contribution in severing inflammation in experimental autoimmune encephalomyelitis (EAE). Matrix metalloprotease-9 (MMP-9) interaction with CD44 has been well known to be involved in cellular adhesion, transmigration and inflammation. In this study, we were interested to examine the role of phagocytic cells expressing MMP-9 in resolving EAE. Materials and Methods: C57BL/6 WT and CD44 KO mice were used as EAE animal model. The level of phagocytic cells expressing MMP-9 in the secondary lymphoid organs were assessed in EAE induced WT as well as CD44 KO animals. Results: EAE severity was found in CD44 KO group compared to WT. Level of CD11b cells (marker of phagocytic cell) in the peritoneal cells expressing MMP-9 was higher in WT compared to CD44 KO. CD11b stained area found to be greater in WT lymph node compared to CD44 KO. Conclusions: This observation suggests the role of CD44 molecule in modulating the immune scenario which is related to disease severity. This study also opens avenues for the specific inflammatory roles of different immune cells in MS.
\end{abstract}

Keywords: multiple sclerosis; EAE; CD44; MMP-9; CD11b; CNS.

\section{INTRODUCTION}

Experimental Autoimmune Encephalomyelitis (EAE) is the primary animal model for multiple sclerosis (MS) research. ${ }^{1}$ The blood brain barrier (BBB) plays a pivotal role in protecting the CNS by preventing entry of unwanted cells and other materials. Proliferation and migration of auto reactive lymphocytes to the CNS is known to be a predominant criteria of EAE. Easy migration of the immune cells is certainly related to the permeability through blood BBB. ${ }^{2}$ CD44 is a novel transmembrane glycoprotein which is able to alter the membrane permeability and its absence severes EAE. $^{3}$ The protective role of CD44 in EAE has been suggested by many researchers in relation to matrix metalloproteases (MMPs), cytokines, chemokines and specific $\mathrm{T}$ lymphocyte populations. ${ }^{3}$

CD44 is known to mediate faster rolling of neutrophils along with macrophages towards the site of infection and inflammation. ${ }^{4} \mathrm{CD} 11 \mathrm{~b}$ is a well -known molecule and expressed mainly on monocytes, macrophages, dendritic cells, neutrophils. ${ }^{5}$ This molecule (CD11b) is frequently used to identify macrophages and microglia. This study suggests that CD44 exerts a protective effect in EAE which is linked to MMP-9+ phagocytic cell increase in the secondary lymphoid organs and probable migration of some specific immune cells to the central nervous system (CNS).

\section{MATERIALS AND METHODS}

Mice and reagents

C57BL/6 wild-type (WT) and CD44-KO mice (The Jackson Laboratory, Bar Harbor) were housed in the animal facilities at Yale University School of Medicine according to Yale University and NIH guidelines.

All mice were used between 8 and 10 weeks of age. Murine MOG peptide was synthesized by the W.M. Keck Biotechnology Resource Laboratory at Yale University (New Haven, CT). Fluorescent conjugated primary antibodies anti-CD11b and antiMMP-9 were purchased from BD Bioscience (San Jose, CA).

\section{EAE Induction}

Mice received $300 \mathrm{mg}$ of rodent $\mathrm{MOG}$ peptide emulsified in complete Freund's adjuvant containing heat-killed Mycobacterium butyricum (Difco, Detroit, MI) via subcutaneous injection on day 0 and

Correspondence: Dr. Sujata Kar, Department of Physiology, College of Medical Sciences-Teaching Hospital, Chitwan, Nepal. Email: sujatakar@hotmail.com. Phone: +977-9811208382. DOI: dx.doi.org/10.3126/jcmsn.v14i3.19381. Article received: 2018-03-13. Article accepted: 2018-08-20. 
7; on day 0 and 2, mice received 500ng of pertussis toxin via intra-peritoneal injection. The mice were monitored daily and graded for clinical symptoms of EAE on the following basis: 0 , no disease; 1,flaccid tail; 2, hind-limb weakness/unsteady gait; 3, hind limb paralysis; 4, hind-and fore-limb weakness/ paralysis; 5 , moribund. ${ }^{3}$

\section{Immunofluorescence and Flow Cytometry}

For immunofluorescence the mice were anesthetized and processed as described. ${ }^{3}$ Six micrometer thick sections of lymph nodes were used for CD11b staining. Before staining, sections were washed with PBS and blocked for 1 hour at room temperature with $1 \%$ bovine serum albumin, 3\% normal goat serum, and $0.3 \%$ Triton $\mathrm{X}-100$ in PBS. Those sections were incubated with ratanti-CD11b (BD Bioscience) for 2 hours at room temperature, followed by goat anti-rat IgG conjugated to Alexafluor-594 (Invitrogen). For flow cytometry, peritoneal cells were collected by peritoneal lavage from WT and CD44 KO mice and prepared for flow cytometry as described. ${ }^{6}$ Staining for $\mathrm{CD} 11 \mathrm{~b}$ and MMP-9 were performed using fluorescently conjugated CD11b (BD Bioscience) and MMP-9 (Santa Cruz) antibodies.

\section{Statistical analysis}

Differences between WT and KO datasets were analyzed using $\mathrm{P}$ values determined by Student's ttest. Error bars represent SEM (standard error of mean).

\section{RESULTS}

Absence of CD44 resulted in severity of EAE

To reconfirm the effect of deletion of CD44 on EAE severity, we assessed the disease scores in WT and CD44 KO mice in each of the set of experiments. We found increased EAE severity in the CD44 KO mice compared to WT mice in each set of experiment (Figure 1).

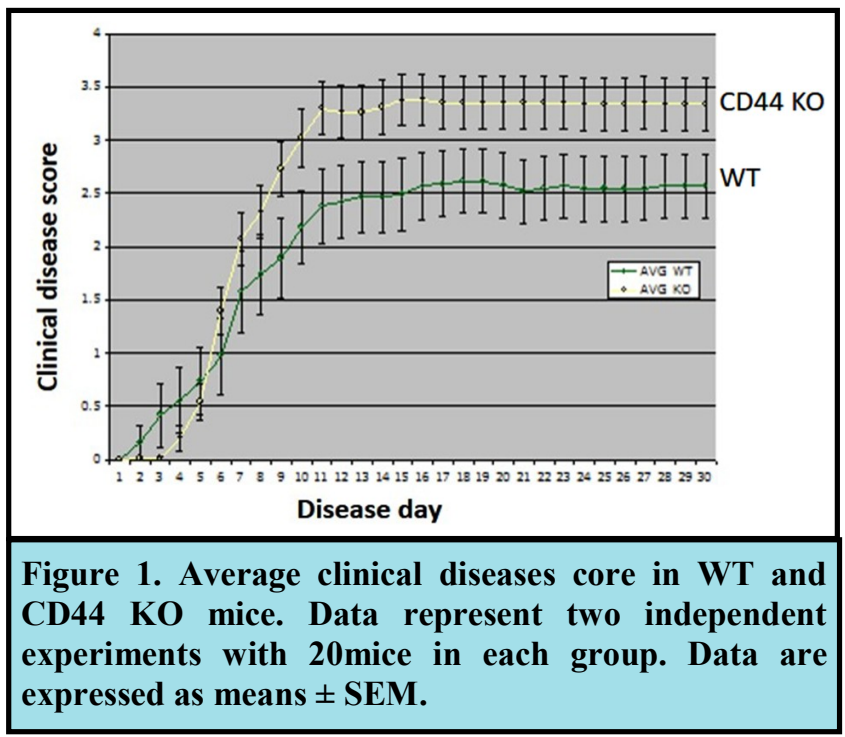

Decrease in the number of CD11b+ cells in the lymph node of CD44 KO mice

To evaluate quantities of phagocytic cells with in the secondary lymphoid organ, lymph nodes were isolated from WT and CD44-KO animals with EAE at the same time point. Immunofluorescent analysis revealed significantly decreased numbers of $\mathrm{CD} 11 \mathrm{~b}+$ (phagocytic cells) cells in the lymph node of the CD44-KO animals compared to WT (Figure 2).

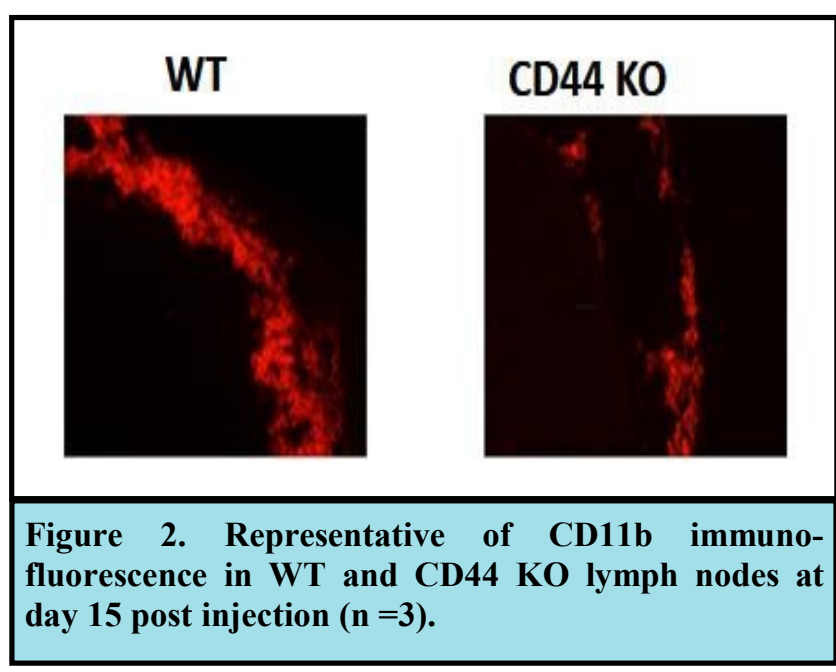

Decrease in the number of MMP-9+ phagocytic cells in the peritoneal lavage fluid of CD44 KO mice

To assess the scenario of MMP-9+ phagocytic cells in the secondary lymphoid organs, we stained the peritoneal cells for CD11b. Those cells were double stained also with MMP-9 antibody. FACS analysis of the peritoneal cells revealed that MMP-9+ phagocytic cells were obviously higher in WT mice compared to CD44 KO ( $\sim 95 \%$ vs $\sim 84 \%$ ) (Figure 3).

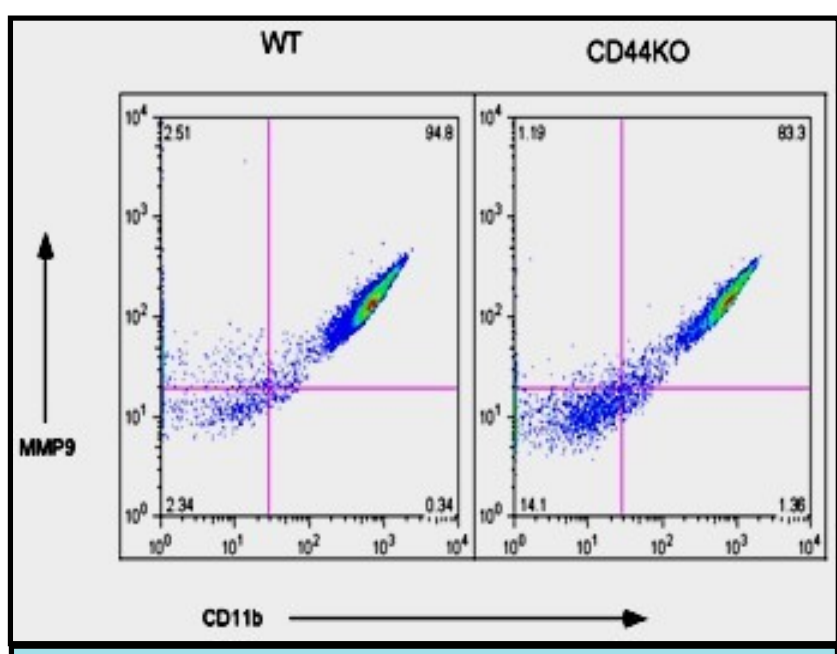

Figure 3. FACS analysis and representative dot plots for CD11b+ MMP-9+ peritoneal cells in WT and CD44 KO naive mice. Data is representative of three independent experiments. 
Kar et al. Increase in MMP-9 Expressing CD11b+ cells in a CD44 Dependent..

\section{DISCUSSION}

EAE, the animal model of MS is involved with disturbance in the body compartments those provide immunity. The pathogenesis of the disease is complex and depends on multiple cell types and processes. ${ }^{7,8}$ The present finding suggests that CD44 provides protective role to some extent in EAE, which is involved withMMP-9 expressing phagocytic cells.

CD44 has already been reported as a negative regulator of inflammation related to T-cell differentiation, adhesion, transendothelial migration and BBB permeability. ${ }^{3}$ CD44 KO junctions are weaker barrier and obviously permeable to the immune cells in an easier way. The weaker barrier in the CD44 KO case is reported to restrict larger molecules. ${ }^{3}$ Increased expression of MMPs in CD44 KO endothelial cells links to weak junctional adhesion, MMP mediated degradation of extracellular matrix and loss of vascular integrity. ${ }^{3}$ This could allow the $\mathrm{T}$ lymphocytes to migrate into the CNS and increase disease severity, ${ }^{9}$ On the other hand, decrease in the number of phagocytic cells in CD44 $\mathrm{KO}$ mice and co-relation of disease severity is meaningful, because phagocytic cells are so important for causing as well as resolving inflammation. ${ }^{10,11}$

\section{CONCLUSIONS}

Higher number of $\mathrm{CD} 11 \mathrm{~b}+$ cells in secondary lymphoid organs (which is a popular marker of phagocytic cells) is obvious in WT mice compared to $\mathrm{CD} 44 \mathrm{KO}$ mice and suggesting the role of phagocytic cells in regulation of the disease severity. These CD11b+ cells were also MMP-9+.

Presence of CD11b+ cells was higher in the lymph node of WT compared to CD44 KO mice. So MMP -9 expressing phagocytic cells probably taking part in the healing of inflammation to some extent which is not happening in CD44 KO mice. It would be of great interest to study and unwind the MMP-9 expression in specific subsets of immune cells in CNS co-related with EAE severity.

\section{ACKNOWLEDGEMENTS}

The entire experimental work was done in Pathology laboratory of Yale University, USA. Data interpretation and manuscript writing were done during our stay in College of Medical Science, Bharatpur, Chitwan, Nepal. We are thankful to Dr. V. Natraj Prasad (Resident Director, COMS), Dr. Manohar Pradhan (Addl. Director) and Dr. Mahesh Mathur (Principal) for their support.
REFERENCES

1. Oliver AR, Lyon GM, Ruddle NH. Rat and human oligodendrocyte glycoproteins induce Experimental Autoimmune Encephalomyelitis by different mechanisms in $\mathrm{C} 57 \mathrm{BL} / 6$ mice. $\mathrm{J}$ Immunol. 2003;171:462.

2. Graesser D, Solowiej A, Bruckner M, Osterweil E, Juedes A, Davis S, et al. Altered vascular permeability and early onset of EAE in PECAM-1 deficient mice. J Clinic Invest. 2002;109:383

3. Flynn KM, Michaud M, Madri JA. CD44 deficiency contributes to enhanced experimental autoimmune encephalomyelitis: arolein immune cells and vascular cells of the blood-brain barrier. The American journal of pathology. 2013;182(4):1322.

4. Riollet C, Rainard P, Poutrel B. Cells and cytokines in inflammatory secretions of bovine mammary gland. Advance Experimental Medical Biology 2000a;480:247.

5. Riollet C, Rainard P, Poutrel B. Cells and cytokines in inflammatory secretions of bovine mammary gland. Advance Experimental Medical Biology 2000b;480:247

6. Graesser D, Mahooti S, Haas T, Davis S, Clark RB, Madri JA. The interrelationship of alpha4 integrin and matrix metalloproteinase- 2 in the pathogenesis of experimental autoimmune encephalomyelitis. Lab Invest. 1998;78:1445

7. Goverman J. Autoimmune Tcell responses in the central nervous system. Nat Rev Immunol.2009;9:393

8. O'Brien K, Fitzgerald DC, Naiken K, Alugupalli KR, Rostami AM, Gran B. Role of the innate immune system in autoimmune inflammatory demyelination. Curr Med Chem. 2008; 15:1105.

9. Guan H, Nagarkatti PS, Nagarkatti M. CD44 reciprocally regulates the differentiation of encephalitogenicTh1/Th17andTh2/regulatory Tcells through epigenetic modulation involving DNA methylation of cytokine gene promoters, thereby controlling the development of experimental autoimmune encephalomyelitis. J Immunol. 2011;186:6955.

10. Thorne RF, Legg JW, Isacke CM. The role of the CD44 transmembrane and cytoplasmic domains in coordinating adhesive and signaling events. J Cell Sci. 2004;117:373.

11. Mikecz K, Brennan FR, Kim JH, Glant TT. Anti -CD44 treatment abrogates tissue oedema and leukocyte infiltration in murine arthritis. Nat Med. 1995; 1:558.

Citation: Kar S, Kar K. Increase in MMP-9 Expressing CD11b+ cells in a CD44 Dependent Way Reduces Severity of Experimental Autoimmune Encephalomyelitis. JCMS Nepal. 2018;14(3):147-9 